\title{
ON THE MEAN OF THE SHIFTED ERROR TERM IN THE THEORY OF THE DIRICHLET DIVISOR PROBLEM
}

\author{
XIAODONG CAO, JUN FURUYA, YOSHIO TANIGAWA \\ AND WENGUANG ZHAI
}

\begin{abstract}
Let $0<\alpha<1$. We show that Bernoulli polynomials appear in the difference $\sum_{n \leq x} \Delta^{j}(n+\alpha)-$ $\int_{1}^{x} \Delta^{j}(t) d t$ for $j=1, \ldots, 4$. As a corollary of this fact, we get better approximations of $\int_{1}^{x} \Delta^{j}(t) d t$ by using zeros of Bernoulli polynomials. For $j=1,2$, we give some interpretation of this fact by means of Dirichlet series with the coefficients $\Delta^{j}(n+\alpha)$.
\end{abstract}

1. Introduction. Let $d(n)$ be the number of positive divisors of $n$, and let $\Delta(n)$ be the error term defined by

$$
\Delta(x)=\sum_{n \leq x} d(n)-x(\log x+2 \gamma-1),
$$

where $\gamma$ is the Euler constant. Many researchers are interested in the behavior of $\Delta(x)$, especially the upper bound and the mean value of powers of $\Delta(x)$. We shall concern with the mean values of $\Delta(x)$ in the discrete and continuous sense.

The first deep result was obtained by Voronoï $[\mathbf{1 6}, \mathbf{1 7}]$. He derived the series representation of $\Delta(x)$ called the Voronoï formula and proved that

$$
\Delta(x) \ll x^{1 / 3+\varepsilon},
$$

where $\varepsilon$ is an arbitrarily small positive constant which need not be the same at each occurrence. The latest result is $\Delta(x) \ll$

2010 AMS Mathematics subject classification. Primary 11M41, 11N37.

Keywords and phrases. Dirichlet divisor problem, mean value formulas, Bernoulli polynomial.

The first and fourth authors are supported by the National Natural Science Foundation of China (grant No. 11171344) and the Natural Science Foundation of Beijing (grant No. 1112010).

Received by the editors on May 18, 2013, and in revised form on April 3, 2014. DOI:10.1216/RMJ-2016-46-1-105 Copyright (C)2016 Rocky Mountain Mathematics Consortium 
$x^{131 / 416}(\log x)^{26947 / 8320}$, which can be found in Huxley [5]. It is conjectured that $\Delta(x) \ll x^{1 / 4+\varepsilon}$.

As for the mean estimate of $\Delta(x)$, Voronoï showed that

$$
\int_{1}^{x} \Delta(t) d t=\frac{1}{4} x+\frac{1}{2 \sqrt{2} \pi^{2}} x^{3 / 4} \sum_{n=1}^{\infty} \frac{d(n)}{n^{5 / 4}} \sin \left(4 \pi \sqrt{n x}-\frac{\pi}{4}\right)+O\left(x^{1 / 4}\right)
$$

and

$$
\sum_{n \leq x} \Delta(n)=\frac{1}{2} x \log x+\left(\gamma-\frac{1}{4}\right) x+O\left(x^{3 / 4}\right)
$$

The mean square estimate of $\Delta(x)$ has a long history $([\mathbf{6}, \mathbf{1 3}])$. Tong $[\mathbf{1 4}]$ proved that

$$
\int_{1}^{x} \Delta^{2}(t) d t=C_{2} x^{3 / 2}+F_{2}(x),
$$

with $F_{2}(x)=O\left(x \log ^{5} x\right)$, where $C_{2}$ is the constant given by $C_{2}=$ $1 / 6 \pi^{2} \sum_{n=1}^{\infty} d^{2}(n) / n^{3 / 2}$. Tong's estimate was improved to $F_{2}(x)=$ $O\left(x \log ^{4} x\right)$ by Preissmann [11] and to $F_{2}(x)=O\left(x \log ^{3} x \log \log x\right)$ by Lau and Tsang [10] recently.

In [9], Lau and Tsang studied the function $F_{2}(x)$ closely and proved that

$$
\int_{2}^{x} F_{2}(t) d t=-\frac{1}{8 \pi^{2}} x^{2} \log ^{2} x+c_{1} x^{2} \log x+O\left(x^{2}\right),
$$

with a certain constant $c_{1}$. Furthermore, they conjectured that

$$
F_{2}(x)=-\frac{1}{4 \pi^{2}} x \log ^{2} x+A_{1} x \log x+O(x),
$$

where $A_{1}$ is a certain constant.

For higher power moments of $\Delta(x)$, it is known that $\int_{1}^{x} \Delta^{j}(t) d t$ $(3 \leq j \leq 9)$ has an asymptotic representation $([\mathbf{1 5}, \mathbf{1 8}, \mathbf{1 9}])$. In particular, in the third power case, the best result to date is due to Ivić and Sargos [8], who proved that

$$
\int_{1}^{x} \Delta^{3}(t) d t=C_{3} x^{7 / 4}+F_{3}(x)
$$


with

$$
F_{3}(x)=O\left(x^{7 / 5+\varepsilon}\right)
$$

where $C_{3}$ is a certain positive constant.

Recently, Furuya [3] studied the difference between the discrete mean $\sum_{n \leq x} \Delta^{j}(n)$ and the continuous mean $\int_{1}^{x} \Delta^{j}(t) d t$ for $j \geq 2$ in detail. For instance, he showed that

$$
\begin{aligned}
\sum_{n \leq x} \Delta(n)= & \int_{1}^{x} \Delta(t) d t+\frac{1}{2} x \log x+\left(\gamma-\frac{1}{2}\right) x \\
& +\left(\frac{1}{2}-\psi(x)\right) \Delta(x)+O(\log x), \\
\sum_{n \leq x} \Delta^{2}(n)= & \int_{1}^{x} \Delta^{2}(t) d t+\frac{1}{6} x \log ^{2} x+\frac{8 \gamma-1}{12} x \log x \\
& +\frac{8 \gamma^{2}-2 \gamma+1}{12} x+O\left(x^{3 / 4} \log x\right)
\end{aligned}
$$

and

$$
\begin{aligned}
\sum_{n \leq x} \Delta^{3}(n)= & \int_{1}^{x} \Delta^{3}(t) d t+\frac{3}{2} C_{2} x^{3 / 2} \log x+(3 \gamma-1) C_{2} x^{3 / 2} \\
& +O\left(x \log ^{5} x\right)
\end{aligned}
$$

where $C_{2}$ is the constant defined by (1.4), and $\psi(x)=x-[x]-1 / 2$.

The error term in (1.9) was studied by Cao and Zhai [2]. They showed that

$$
\begin{aligned}
\sum_{n \leq x} \Delta^{2}(n)= & \int_{1}^{x} \Delta^{2}(t) d t+\frac{1}{6} x \log ^{2} x \\
& +\frac{8 \gamma-1}{12} x \log x+\frac{8 \gamma^{2}-2 \gamma+1}{12} x \\
& +(\log x+2 \gamma) G(x)+\left(\frac{1}{2}-\psi(x)\right) \Delta^{2}(x)+O\left(x^{1 / 2} \log x\right)
\end{aligned}
$$


with

$$
G(x)=\frac{1}{2 \sqrt{2} \pi^{2}} x^{3 / 4} \sum_{n=1}^{\infty} \frac{d(n)}{n^{5 / 4}} \sin \left(4 \pi \sqrt{n x}-\frac{\pi}{4}\right)
$$

In [1], with a different method from [3], the authors of the present paper showed

$$
\begin{aligned}
\sum_{n \leq x} \Delta^{4}(n)= & \int_{1}^{x} \Delta^{4}(t) d t+2 C_{3} x^{7 / 4} \log x \\
& +\frac{4(7 \gamma-2)}{7} C_{3} x^{7 / 4}+C_{2} x^{3 / 2} \log ^{2} x \\
& +\frac{4(3 \gamma-1)}{3} C_{2} x^{3 / 2} \log x+\frac{4\left(9 \gamma^{2}-6 \gamma+2\right)}{9} C_{2} x^{3 / 2} \\
& +O\left(x^{7 / 5+\varepsilon}\right)
\end{aligned}
$$

where $C_{2}$ is the same as above and $C_{3}$ is the constant defined by (1.6).

We can observe that the difference between the discrete mean of $\Delta^{j}(n)$ and the continuous mean of $\Delta^{j}(x)$ is rather large. The reason may be that $\Delta(x)$ has a jump at each integer $n$. This remark suggests that we take a sum at non-integer points. In this paper, we shall consider the sum of the form $\sum_{n \leq x} \Delta^{j}(n+\alpha)$, where $\alpha$ is a constant such that $0<\alpha<1$. As there is a lack of precise form of the discrete and continuous mean of higher powers of $\Delta(x)$ at present, we restrict ourselves to consideration of up to the fourth power moments.

Theorem 1.1. Let $\alpha$ be a constant such that $0 \leq \alpha<1$. Let $B_{1}(x)=x-1 / 2$ and $B_{2}(x)=x^{2}-x+1 / 6$ be the Bernoulli polynomials of degree 1 and 2, respectively. Then, under the above notation, we have

$$
\begin{aligned}
\sum_{n \leq x} \Delta(n+\alpha)= & \int_{1}^{x} \Delta(t) d t-B_{1}(\alpha) x(\log x+2 \gamma-1) \\
& +\left(\frac{1}{2}-\psi(x)\right) \Delta(x)+O(\log x)
\end{aligned}
$$




$$
\begin{aligned}
\sum_{n \leq x} \Delta^{2}(n+\alpha)= & \int_{1}^{x} \Delta^{2}(t) d t+B_{2}(\alpha) x \log ^{2} x \\
& +\left((4 \gamma-2) B_{2}(\alpha)-\frac{1}{2} B_{1}(\alpha)\right) x \log x \\
& +\left(\left(4 \gamma^{2}-4 \gamma+2\right) B_{2}(\alpha)-\left(\gamma-\frac{1}{2}\right) B_{1}(\alpha)\right) x \\
& -2 B_{1}(\alpha)(\log x+2 \gamma) G(x)+\left(\frac{1}{2}-\psi(x)\right) \Delta^{2}(x) \\
& +O\left(x^{1 / 2} \log x\right) .
\end{aligned}
$$

$$
\begin{aligned}
\sum_{n \leq x} \Delta^{3}(n+\alpha)= & \int_{1}^{x} \Delta^{3}(t) d t-3 B_{1}(\alpha) C_{2} x^{3 / 2} \log x \\
& -(6 \gamma-2) B_{1}(\alpha) C_{2} x^{3 / 2}+O\left(x \log ^{5} x\right) .
\end{aligned}
$$

and

$$
\begin{aligned}
\sum_{n \leq x} \Delta^{4}(n+\alpha)= & \int_{1}^{x} \Delta^{4}(t) d t-4 C_{3} B_{1}(\alpha) x^{7 / 4} \log x \\
& -\frac{8}{7}(7 \gamma-2) C_{3} B_{1}(\alpha) x^{7 / 4} \\
& +6 C_{2} B_{2}(\alpha) x^{3 / 2} \log ^{2} x+8(3 \gamma-1) C_{2} B_{2}(\alpha) x^{3 / 2} \log x \\
& +8\left(3 \gamma^{2}-2 \gamma+\frac{2}{3}\right) C_{2} B_{2}(\alpha) x^{3 / 2}+O\left(x^{7 / 5+\varepsilon}\right) .
\end{aligned}
$$

The more precise form of (1.16) is derived under the conjecture of Lau and Tsang (1.5). In fact, we can prove that

Theorem 1.2. Suppose that the conjecture of Lau and Tsang (1.5) is true. Let $B_{3}(x)=x^{3}-3 / 2 x^{2}+1 / 2 x$ be the Bernoulli polynomial of degree 3 . Then we have

$$
\sum_{n \leq x} \Delta^{3}(n+\alpha)=\int_{1}^{x} \Delta^{3}(t) d t-3 C_{2} B_{1}(\alpha) x^{3 / 2} \log x
$$




$$
\begin{aligned}
& -2 C_{2}(3 \gamma-1) B_{1}(\alpha) x^{3 / 2} \\
& -\left\{B_{3}(\alpha)-\frac{3}{4 \pi^{2}} B_{1}(\alpha)\right\} x \log ^{3} x \\
& +3\left\{(1-2 \gamma) B_{3}(\alpha)+\frac{1}{4} B_{2}(\alpha)\right. \\
& \left.-\left(\frac{(1-2 \gamma)}{4 \pi^{2}}+A_{1}\right) B_{1}(\alpha)\right\} x \log ^{2} x+O(x \log x) .
\end{aligned}
$$

It is interesting to note that the Bernoulli polynomials appear in the coefficients on the right-hand sides of Theorems 1.1 and 1.2. Hence, if we take $\alpha$ as a root of $B_{1}(x)$ or $B_{2}(x)$, we can get better approximations of $\int_{0}^{x} \Delta^{j}(t) d t$. In fact, in Section 5 , we shall consider the sum of the forms $\sum_{n \leq x} \Delta^{j}(n+1 / 2)$ or $\sum_{n \leq x}\left(\Delta^{j}(n+\beta)+\Delta^{j}\left(n+\beta^{\prime}\right)\right) / 2$ to get such approximations, where $\beta$ and $\beta^{\prime}$ are zeros of $B_{2}(x)$.

It is well known that the behavior of the sum of an arithmetical function is controlled by the singularities of the Dirichlet series generated by that arithmetical function. Hence, the assertions of Theorem 1.1 could be interpreted by means of the Dirichlet series $\sum_{n=1}^{\infty} \Delta^{j}(n+\alpha) / n^{s}$. In the last section, we shall discuss Theorem 1.1 from this viewpoint.

2. Preliminaries. First we shall give an expression of $\Delta(n+\alpha)$ by means of $\Delta(n)$.

Lemma 2.1. Let $\alpha$ be a real number such that $0 \leq \alpha<1$ and $n a$ positive integer. Then we have

$$
\Delta(n+\alpha)=\Delta(n)-\alpha \log n-2 \alpha \gamma-\frac{\alpha^{2}}{2 n}+O\left(\frac{1}{n^{2}}\right) .
$$

Proof. From the definition of $\Delta(n)$, we have

$$
\Delta(n)+n(\log n+2 \gamma-1)=\Delta(n+\alpha)+(n+\alpha)(\log (n+\alpha)+2 \gamma-1) .
$$

Hence,

$$
\Delta(n+\alpha)=\Delta(n)-\left((n+\alpha) \log \left(1+\frac{\alpha}{n}\right)+\alpha \log n\right)-\alpha(2 \gamma-1)
$$




$$
\begin{aligned}
= & \Delta(n)-\sum_{k=1}^{N} \frac{(-1)^{k-1}}{k(k+1)} \frac{\alpha^{k+1}}{n^{k}}-\alpha \log n \\
& -2 \alpha \gamma+O\left(\frac{1}{n^{N+1}}\right),
\end{aligned}
$$

where $N$ is any positive integer. Taking $N=1$ in (2.2), we get (2.1).

In this place, we shall collect the formulas for the sum of powers of $\log n$ for later use. The proofs will be omitted since they are elementary and well known.

Lemma 2.2. We have, for $x \geq 2$,

$$
\begin{aligned}
& \sum_{n \leq x} \log n=x \log x-x+O(\log x), \\
& \sum_{n \leq x} \log ^{2} n=x \log ^{2} x-2(x \log x-x)+O\left(\log ^{2} x\right), \\
& \sum_{n \leq x} \log ^{3} n=x \log ^{3} x-3 x \log ^{2} x+6 x \log x-6 x+O\left(\log ^{3} x\right), \\
& \sum_{n \leq x} \frac{\log n}{n} \ll \log ^{2} x .
\end{aligned}
$$

\section{Proof of Theorem 1.1.}

Proof of (1.14). From Lemma 2.1, we have

$$
\sum_{n \leq x} \Delta(n+\alpha)=\sum_{n \leq x} \Delta(n)-\alpha \sum_{n \leq x} \log n-2 \alpha \gamma[x]+O(\log x) .
$$

Substituting (1.8) in (3.1) and using Lemma 2.2, we get (1.14) immediately.

Proof of (1.15). Squaring both sides of (2.1) and noting (1.1), we get

$$
\begin{aligned}
\Delta^{2}(n+\alpha)= & \Delta^{2}(n)-2 \alpha \Delta(n) \log n-4 \alpha \gamma \Delta(n)-\frac{\alpha^{2} \Delta(n)}{n} \\
& +\alpha^{2} \log ^{2} n+4 \alpha^{2} \gamma \log n+4 \alpha^{2} \gamma^{2}+\frac{\alpha^{3} \log n}{n} \\
& +\frac{2 \alpha^{3} \gamma}{n}+O\left(n^{-5 / 3}\right) .
\end{aligned}
$$


It is easily seen that

$$
\sum_{n \leq x} \frac{\Delta(n)}{n} \ll \log ^{2} x
$$

by partial summation and (1.3), and

$$
\begin{aligned}
\sum_{n \leq x} \Delta(n)= & \frac{1}{2} x \log x+\left(\gamma-\frac{1}{4}\right) x+G(x) \\
& +\left(\frac{1}{2}-\psi(x)\right) \Delta(x)+O\left(x^{1 / 4}\right)
\end{aligned}
$$

by (1.2) and (1.8).

Next, we show that

$$
\begin{aligned}
\sum_{n \leq x} \Delta(n) \log n= & \frac{1}{2} x \log ^{2} x+\left(\gamma-\frac{3}{4}\right) x \log x \\
& -\left(\gamma-\frac{3}{4}\right) x+G(x) \log x \\
& +\left(\frac{1}{2}-\psi(x)\right) \Delta(x) \log x+O\left(x^{1 / 4} \log x\right) .
\end{aligned}
$$

In fact, by partial summation and (1.8), we have

$$
\begin{aligned}
\sum_{n \leq x} \Delta(n) \log n= & \log x\left(\sum_{n \leq x} \Delta(n)\right)-\int_{1}^{x} \frac{1}{t}\left(\sum_{n \leq t} \Delta(n)\right) d t \\
= & \frac{1}{2} x \log ^{2} x+\left(\gamma-\frac{3}{4}\right) x \log x \\
& -\left(\gamma-\frac{3}{4}\right) x+G(x) \log x \\
& +\left(\frac{1}{2}-\psi(x)\right) \Delta(x) \log x-\int_{1}^{x} t^{-1} G(t) d t \\
& -\int_{1}^{x}\left(\frac{1}{2}-\psi(t)\right) \frac{\Delta(t)}{t} d t+O\left(x^{1 / 4} \log x\right) .
\end{aligned}
$$

As for the sixth term on the right-hand side of (3.6), one may integrate term by term, since the series of $G(x)$ is absolutely convergent. Hence, 
we get

$$
\begin{aligned}
\int_{1}^{x} t^{-1} G(t) d t & =\frac{1}{2 \sqrt{2} \pi^{2}} \sum_{n=1}^{\infty} \frac{d(n)}{n^{5 / 4}} \int_{1}^{x} t^{-1 / 4} \sin \left(4 \pi \sqrt{n t}-\frac{\pi}{4}\right) d t \\
& \ll \sum_{n=1}^{\infty} \frac{d(n)}{n^{5 / 4}} \frac{x^{1 / 4}}{\sqrt{n}} \ll x^{1 / 4} .
\end{aligned}
$$

Here we used the first derivative test for the integral on the right-hand side.

For the seventh term in the right-hand side of (3.6), we apply Cauchy's inequality and (1.4) to get

$$
\begin{aligned}
\int_{X}^{2 X}\left(\frac{1}{2}-\psi(t)\right) \frac{\Delta(t)}{t} d t & \ll\left(\int_{X}^{2 X} \Delta^{2}(t) d t\right)^{1 / 2}\left(\int_{X}^{2 X} t^{-2} d t\right)^{1 / 2} \\
& \ll X^{1 / 4} .
\end{aligned}
$$

Hence, by the splitting argument

$$
\int_{1}^{x}\left(\frac{1}{2}-\psi(t)\right) \frac{\Delta(t)}{t} d t \ll x^{1 / 4} .
$$

The sixth and seventh terms are absorbed into the error term, and this completes the proof of (3.5).

Now take the sum over $n$ on both sides of (3.2). Using Lemma 2.2, (3.3), (3.4) and (3.5), we find that

$$
\begin{aligned}
& \sum_{n \leq x} \Delta^{2}(n+\alpha) \\
& =\sum_{n \leq x} \Delta^{2}(n)+\left(\alpha^{2}-\alpha\right) x \log ^{2} x+\left((4 \gamma-2) \alpha^{2}-\left(4 \gamma-\frac{3}{2}\right) \alpha\right) x \log x \\
& \quad+\left(\left(4 \gamma^{2}-4 \gamma+2\right) \alpha^{2}+\left(-4 \gamma^{2}+3 \gamma-\frac{3}{2}\right) \alpha\right) x \\
& \quad-2 \alpha(\log x+2 \gamma)\left(G(x)+\left(\frac{1}{2}-\psi(x)\right) \Delta(x)\right)+O\left(x^{1 / 4} \log x\right)
\end{aligned}
$$

Substituting (1.11) in the right hand side of (3.8) and using (1.1), we 
find that

$$
\begin{aligned}
\sum_{n \leq x} \Delta^{2}(n+\alpha)= & \int_{1}^{x} \Delta^{2}(t) d t+\left(\alpha^{2}-\alpha+\frac{1}{6}\right) x \log ^{2} x \\
& +\left(4 \gamma\left(\alpha^{2}-\alpha+\frac{1}{6}\right)-2\left(\alpha^{2}-\frac{3}{4} \alpha+\frac{1}{24}\right)\right) x \log x \\
& +\left(4 \gamma^{2}\left(\alpha^{2}-\alpha+\frac{1}{6}\right)-(4 \gamma-2)\left(\alpha^{2}-\frac{3}{4} \alpha+\frac{1}{24}\right)\right) x \\
& +(1-2 \alpha)(\log x+2 \gamma) G(x) \\
& +\left(\frac{1}{2}-\psi(x)\right) \Delta^{2}(x)+O\left(x^{1 / 2} \log x\right)
\end{aligned}
$$

which proves (1.15).

Remark. The inequality (3.7) can be improved to $\ll \log x$ with the help of (1.2) and the similar discussion given in [3, pages 16-18], but the above estimate is enough for our purposes.

Proof of (1.16). Taking the third power of both sides of (2.1), we have

$$
\begin{aligned}
\Delta^{3}(n+\alpha)= & \Delta^{3}(n)-3 \alpha \Delta^{2}(n) \log n-6 \alpha \gamma \Delta^{2}(n) \\
& +3 \alpha^{2} \Delta(n) \log ^{2} n+12 \alpha^{2} \gamma \Delta(n) \log n \\
& +12 \alpha^{2} \gamma^{2} \Delta(n)-\frac{3}{2} \alpha^{2} \frac{\Delta^{2}(n)}{n} \\
& +3 \alpha^{3} \frac{\Delta(n) \log n}{n}+6 \alpha^{3} \gamma \frac{\Delta(n)}{n}-\alpha^{3} \log ^{3} n \\
& -6 \alpha^{3} \gamma \log ^{2} n+O(\log n) .
\end{aligned}
$$

Similarly to (3.5), we find that

$$
\begin{aligned}
\sum_{n \leq x} \Delta(n) \log ^{2} n= & \frac{1}{2} x \log ^{3} x+\left(\gamma-\frac{5}{4}\right) x \log ^{2} x \\
& -\left(2 \gamma-\frac{5}{2}\right)(x \log x-x) \\
& +G(x) \log ^{2} x+\left(\frac{1}{2}-\psi(x)\right) \Delta(x) \log ^{2} x
\end{aligned}
$$




$$
\begin{gathered}
\quad+O\left(x^{1 / 4} \log ^{2} x\right) \\
=\frac{1}{2} x \log ^{3} x+\left(\gamma-\frac{5}{4}\right) x \log ^{2} x \\
-\left(2 \gamma-\frac{5}{2}\right) x \log x+O(x) .
\end{gathered}
$$

Furthermore, it is easy to see that

$$
\sum_{n \leq x} \frac{\Delta^{2}(n)}{n} \ll x^{1 / 2}
$$

and

$$
\sum_{n \leq x} \frac{\Delta(n) \log n}{n} \ll \log ^{3} x
$$

By making use of (1.3), (3.3), (3.6), (3.11)-(3.13) and Lemma 2.2, we take the sum over $n$ on both sides of (3.10) and get

$$
\begin{aligned}
\sum_{n \leq x} \Delta^{3}(n+\alpha)= & \sum_{n \leq x} \Delta^{3}(n)-3 \alpha \sum_{n \leq x} \Delta^{2}(n) \log n \\
& -6 \alpha \gamma \sum_{n \leq x} \Delta^{2}(n)+\left(\frac{3}{2} \alpha^{2}-\alpha^{3}\right) x \log ^{3} x \\
& +\left(3 \alpha^{2}\left(3 \gamma-\frac{5}{4}\right)+3 \alpha^{3}(1-2 \gamma)\right) x \log ^{2} x \\
& +O(x \log x) .
\end{aligned}
$$

As for the second and the third sums on the right hand side of (3.14), from (1.4) and (1.9), we have

$$
\sum_{n \leq x} \Delta^{2}(n)=C_{2} x^{3 / 2}+O\left(x \log ^{4} x\right),
$$

(here we have used Preissmann's estimate of $F(x)$ for simplicity), and hence by partial summation,

$$
\sum_{n \leq x} \Delta^{2}(n) \log n=\log x\left(C_{2} x^{3 / 2}+O\left(x \log ^{4} x\right)\right)
$$




$$
\begin{aligned}
& -\int_{1}^{x} \frac{1}{t}\left(C_{2} t^{3 / 2}+O\left(t \log ^{4} t\right)\right) d t \\
= & C_{2} x^{3 / 2} \log x-\frac{2}{3} C_{2} x^{3 / 2}+O\left(x \log ^{5} x\right) .
\end{aligned}
$$

Therefore, the left hand side of (3.14) (by the error estimate $O\left(x \log ^{5} x\right)$ ) is expressed as

$$
\begin{aligned}
\sum_{n \leq x} \Delta^{3}(n+\alpha)= & \sum_{n \leq x} \Delta^{3}(n)-3 \alpha C_{2} x^{3 / 2} \log x \\
& +2 \alpha(1-3 \gamma) C_{2} x^{3 / 2}+O\left(x \log ^{5} x\right) .
\end{aligned}
$$

Now, combining (1.10) and the above equation, we get

$$
\begin{aligned}
\sum_{n \leq x} \Delta^{3}(n+\alpha)= & \int_{1}^{x} \Delta^{3}(t) d t+3\left(\frac{1}{2}-\alpha\right) C_{2} x^{3 / 2} \log x \\
& +(3 \gamma-1)(1-2 \alpha) C_{2} x^{3 / 2}+O\left(x \log ^{5} x\right) .
\end{aligned}
$$

This completes the proof of (1.16).

Proof of (1.17). Taking the fourth power of (2.1), we have

$$
\begin{aligned}
\Delta^{4}(n+\alpha)= & \Delta^{4}(n)-4 \alpha \Delta^{3}(n) \log n-8 \alpha \gamma \Delta^{3}(n) \\
& +6 \alpha^{2} \Delta^{2}(n) \log ^{2} n+24 \alpha^{2} \gamma \Delta^{2}(n) \log n \\
& +24 \alpha^{2} \gamma^{2} \Delta^{2}(n)-4 \alpha^{3} \Delta(n) \log ^{3} n \\
& -24 \alpha^{3} \gamma \Delta(n) \log ^{2} n-48 \alpha^{3} \gamma^{2} \Delta(n) \log n \\
& -32 \alpha^{3} \gamma^{3} \Delta(n)+O\left(\log ^{4} n\right) .
\end{aligned}
$$

From (1.6), (1.7) and (1.10), we see that

$$
\begin{aligned}
\sum_{n \leq x} \Delta^{3}(n)= & C_{3} x^{7 / 4}+\frac{3}{2} C_{2} x^{3 / 2} \log x+(3 \gamma-1) C_{2} x^{3 / 2} \\
& +O\left(x^{7 / 5+\varepsilon}\right)
\end{aligned}
$$

hence by partial summation, we get

$$
\sum_{n \leq x} \Delta^{3}(n) \log n=C_{3} x^{7 / 4} \log x-\frac{4}{7} C_{3} x^{7 / 4}
$$




$$
\begin{aligned}
& +\frac{3}{2} C_{2} x^{3 / 2} \log ^{2} x+(3 \gamma-2) C_{2} x^{3 / 2} \log x \\
& -\frac{2}{3}(3 \gamma-2) C_{2} x^{3 / 2}+O\left(x^{7 / 5+\varepsilon}\right) .
\end{aligned}
$$

We also need the following estimate:

$$
\begin{aligned}
\sum_{n \leq x} \Delta^{2}(n) \log ^{2} n= & C_{2} x^{3 / 2} \log ^{2} x-\frac{4}{3} C_{2} x^{3 / 2} \log x \\
& +\frac{8}{9} C_{2} x^{3 / 2}+O\left(x \log ^{6} x\right) .
\end{aligned}
$$

The sums arising from the terms after the seventh one in the right hand side of (3.18) are estimated as $O\left(x \log ^{4} x\right)$.

By using (3.15), (3.16) and (3.19)-(3.21), we make the sum over $n$ on both sides of (3.18). This time, we have

$$
\begin{aligned}
\sum_{n \leq x} \Delta^{4}(n+\alpha)= & \sum_{n \leq x} \Delta^{4}(n)-4 \alpha \sum_{n \leq x} \Delta^{3}(n) \log n-8 \alpha \gamma \sum_{n \leq x} \Delta^{3}(n) \\
& +6 \alpha^{2} \sum_{n \leq x} \Delta^{2}(n) \log ^{2} n+24 \alpha^{2} \gamma \sum_{n \leq x} \Delta^{2}(n) \log n \\
& +24 \alpha^{2} \gamma^{2} \sum_{n \leq x} \Delta^{2}(n)+O\left(x \log ^{4} x\right) \\
= & \sum_{n \leq x} \Delta^{4}(n)-4 \alpha C_{3} x^{7 / 4} \log x+\left(\frac{16}{7}-8 \gamma\right) \alpha C_{3} x^{7 / 4} \\
& +6\left(\alpha^{2}-\alpha\right) C_{2} x^{3 / 2} \log ^{2} x \\
& +8(3 \gamma-1)\left(\alpha^{2}-\alpha\right) C_{2} x^{3 / 2} \log x \\
& +8\left(3 \gamma^{2}-2 \gamma+\frac{2}{3}\right)\left(\alpha^{2}-\alpha\right) C_{2} x^{3 / 2}+O\left(x^{7 / 5+\varepsilon}\right) .
\end{aligned}
$$

Therefore, by (1.13), we deduce that

$$
\begin{aligned}
\sum_{n \leq x} \Delta^{4}(n+\alpha)= & \int_{1}^{x} \Delta^{4}(t) d t+2 C_{3}(1-2 \alpha) x^{7 / 4} \log x \\
& +\frac{4}{7}(7 \gamma-2)(1-2 \alpha) C_{3} x^{7 / 4}
\end{aligned}
$$




$$
\begin{aligned}
& +6\left(\alpha^{2}-\alpha+\frac{1}{6}\right) C_{2} x^{3 / 2} \log ^{2} x \\
& +8(3 \gamma-1)\left(\alpha^{2}-\alpha+\frac{1}{6}\right) C_{2} x^{3 / 2} \log x \\
& +8\left(3 \gamma^{2}-2 \gamma+\frac{2}{3}\right)\left(\alpha^{2}-\alpha+\frac{1}{6}\right) C_{2} x^{3 / 2}+O\left(x^{7 / 5+\varepsilon}\right)
\end{aligned}
$$

This proves (1.17).

4. Proof of Theorem 1.2. We assume that the conjecture (1.5) of Lau and Tsang is true. Under this assumption, Furuya [3, Theorem 2] proved that

$$
\begin{aligned}
\sum_{n \leq x} \Delta^{3}(n)= & \int_{1}^{x} \Delta^{3}(t) d t+\frac{3}{2} C_{2} x^{3 / 2} \log x+(3 \gamma-1) C_{2} x^{3 / 2} \\
& -\frac{3}{8 \pi^{2}} x \log ^{3} x \\
& +\left(\frac{3}{8 \pi^{2}}-\frac{3}{4 \pi^{2}} \gamma+\frac{1}{8}+\frac{3}{2} A_{1}\right) x \log ^{2} x+O(x \log x)
\end{aligned}
$$

where $A_{1}$ is the constant which appears in Lau and Tsang's conjecture (1.5).

Furthermore, their conjecture (1.5) with (1.9) and (1.4) gives

$$
\begin{aligned}
\sum_{n \leq x} \Delta^{2}(n)= & C_{2} x^{3 / 2}+\left(\frac{1}{6}-\frac{1}{4 \pi^{2}}\right) x \log ^{2} x \\
& +\left(A_{1}+\frac{8 \gamma-1}{12}\right) x \log x+O(x),
\end{aligned}
$$

and

$$
\begin{aligned}
\sum_{n \leq x} \Delta^{2}(n) \log n= & C_{2} x^{3 / 2} \log x-\frac{2}{3} C_{2} x^{3 / 2} \\
& +\left(\frac{1}{6}-\frac{1}{4 \pi^{2}}\right) x \log ^{3} x \\
& +\left(A_{1}+\frac{8 \gamma-1}{12}-\left(\frac{1}{6}-\frac{1}{4 \pi^{2}}\right)\right) x \log ^{2} x+O(x \log x) .
\end{aligned}
$$


by partial summation. Therefore, from (3.14) and (4.1)-(4.3), we obtain

$$
\begin{aligned}
\sum_{n \leq x} \Delta^{3}(n+\alpha)= & \int_{1}^{x} \Delta^{3}(t) d t+3 C_{2}\left(\frac{1}{2}-\alpha\right) x^{3 / 2} \log x \\
& +C_{2}(3 \gamma-1)(1-2 \alpha) x^{3 / 2} \\
& +\left\{3\left(\frac{1}{2}-\alpha\right)\left(\frac{1}{6}-\frac{1}{4 \pi^{2}}\right)-\frac{1}{4}+\frac{3}{2} \alpha^{2}-\alpha^{3}\right\} x \log ^{3} x \\
& +\left\{\left(\frac{3(1-2 \gamma)}{8 \pi^{2}}+\frac{3}{2} A_{1}\right)(1-2 \alpha)+\frac{1}{8}+\frac{3}{4} \alpha-3 \alpha \gamma\right. \\
& \left.+3 \alpha^{2}\left(3 \gamma-\frac{5}{4}\right)+3 \alpha^{3}(1-2 \gamma)\right\} x \log ^{2} x+O(x \log x) .
\end{aligned}
$$

Noting that $B_{3}(x)=x^{3}-3 / 2 x^{2}+1 / 2$, we get the assertion of Theorem 1.2.

5. Better approximation for the integral of $\Delta^{j}(x)$. As we remarked in Section $1, \sum_{n \leq x} \Delta^{j}(n)$ does not give a good approximation for $\int_{0}^{x} \Delta^{j}(t) d t$. But, by specifying $\alpha$ in Theorems 1.1 and 1.2 , we can get better approximations. Namely, we have

Corollary 5.1. We have

$$
\sum_{n \leq x} \Delta\left(n+\frac{1}{2}\right)=\int_{1}^{x} \Delta(t) d t+\left(\frac{1}{2}-\psi(x)\right) \Delta(x)+O(\log x)
$$

and

$$
\sum_{n \leq x} \Delta^{3}\left(n+\frac{1}{2}\right)=\int_{1}^{x} \Delta^{3}(t) d t+O\left(x \log ^{5} x\right)
$$

Corollary 5.2. Let $\beta$ and $\beta^{\prime}$ be zeros of the Bernoulli polynomial $B_{2}(x)$ of degree 2. Then we have

$$
\begin{aligned}
& \sum_{n \leq x} \frac{\Delta^{2}(n+\beta)+\Delta^{2}\left(n+\beta^{\prime}\right)}{2} \\
& \quad=\int_{1}^{x} \Delta^{2}(t) d t+\left(\frac{1}{2}-\psi(x)\right) \Delta^{2}(x)+O\left(x^{1 / 2} \log x\right),
\end{aligned}
$$




$$
\sum_{n \leq x} \frac{\Delta^{3}(n+\beta)+\Delta^{3}\left(n+\beta^{\prime}\right)}{2}=\int_{1}^{x} \Delta^{3}(t) d t+O\left(x \log ^{5} x\right),
$$

and

$$
\sum_{n \leq x} \frac{\Delta^{4}(n+\beta)+\Delta^{4}\left(n+\beta^{\prime}\right)}{2}=\int_{1}^{x} \Delta^{4}(t) d t+O\left(x^{7 / 5+\varepsilon}\right) .
$$

Since $B_{3}(x)=B_{1}(x)\left(B_{2}(x)-1 / 6\right)$, we get from Theorem 1.2 that

Corollary 5.3. Let $\beta$ and $\beta^{\prime}$ be zeros of $B_{2}(x)$. Assume that the Lau and Tsang conjecture (1.5) is true. Then, we have

$$
\sum_{n \leq x} \frac{\Delta^{3}(n+\beta)+\Delta^{3}\left(n+\beta^{\prime}\right)}{2}=\int_{1}^{x} \Delta^{3}(t) d t+O(x \log x) .
$$

It is interesting to note that, under the conjecture of Lau and Tsang, the error term in (5.3) is improved to $O(x \log x)$ in (5.4). There seems to be no conjecture on the behavior for $F_{3}(x)$ in (1.6) similar to (1.5).

6. Dirichlet series of $\Delta^{j}(n+\alpha)$. In this section, we give some interpretation of Corollaries 5.1 and 5.2 from the viewpoint of the Dirichlet series. Let $\mathcal{D}(s)$ be a Dirichlet series, which is first defined by the absolutely convergent series $\sum_{n=1}^{\infty} a_{n} n^{-s}$ and continued as a meromorphic function to some right-half plane. Let $\sum_{n \leq x} a_{n}=$ $g(x)+E(x)$, where $g(x)$ is the main term and $E(x)$ is the error term. It is expected that $g(x)$ is obtained from the residues of the poles of $\mathcal{D}(s) x^{s} / s$. For a rigorous proof, we need more precise information on the order of gratitude of $\mathcal{D}(s)$ as $|\Im s| \rightarrow \infty$. Here, we shall see that the assertions in Corollaries 5.1 and 5.2 are compatible with the above heuristic argument. See also the last section of [4].

Let $D_{j}(s, \alpha)$ and $I_{j}(s)$ be functions defined by

$$
D_{j}(s, \alpha)=\sum_{n=1}^{\infty} \frac{\Delta^{j}(n+\alpha)}{n^{s}}
$$


and

$$
I_{j}(s)=\int_{1}^{\infty} t^{-s} \Delta^{j}(t) d t
$$

respectively. We simply write $D_{j}(s)=D_{j}(s, 0)$. The analytic properties of $D_{j}(s)$ and $I_{j}(s)$ are known in the cases $j=1,2$ [4], but unfortunately we know little about them in the case $j \geq 3$. So we shall restrict ourselves to the cases $j=1,2$. Equations (5.1) and (5.2) suggest that $D_{1}(s, 1 / 2)-L_{1}(s)$ and $\left[D_{2}(s, \beta)+D_{2}\left(s, \beta^{\prime}\right)\right] / 2-I_{2}(s)$ are holomorphic for $\Re s>1-c$ with some constant $c>0$, which we will show now.

We recall some facts from [4]. For $j=1,2, D_{j}(s, \alpha)$ and $I_{j}(s)$ converges absolutely in the range $\Re s>1+j / 4$. It is shown that $D_{1}(s)$ can be continued analytically to the whole plane $\mathbb{C}$ as a meromorphic function. It has a double pole at $s=1$ and simple poles at $s=-2 n$ $(n=0,1,2, \ldots)$. In particular, the Laurent expansion of $D_{1}(s)$ at $s=1$ is given by

$$
D_{1}(s)=\frac{1}{2(s-1)^{2}}+\frac{\gamma+1 / 4}{s-1}+O(1)
$$

(see $\left[4\right.$, Theorem 1]). The function $I_{1}(s)$ can be expressed as

$$
I_{1}(s)=\frac{\zeta^{2}(s-1)}{s-1}-\frac{2 \gamma-1}{s-2}-\frac{1}{(s-2)^{2}},
$$

(see Sitaramachandrarao [12]). Note that $s=2$ is not a pole of $I_{1}(s)$.

On the other hand, it is shown in [4] that $D_{2}(s)$ and $I_{2}(s)$ can be analytically continued to the region $\Re s>2 / 3$ as meromorphic functions [4, Theorem 2]. They have a simple pole at $s=3 / 2$ with the same residue and a triple pole at $s=1$. The results for $I_{2}(s)$ are essentially due to Ivić [7]. (See also [4, Lemmas 3 and 4].) Furthermore, we showed that $D_{2}(s)-I_{2}(s)$ has an analytic continuation to the region $\Re s>1 / 2$ with the triple pole at $s=1$, whose Laurent expansion at $s=1$ is given by

$$
\begin{aligned}
D_{2}(s)-I_{2}(s)= & \frac{1}{3(s-1)^{3}}+\frac{2 / 3 \gamma+1 / 4}{(s-1)^{2}} \\
& +\frac{(2 / 3 \gamma+1 / 2) \gamma}{s-1}+O(1) .
\end{aligned}
$$


We return to the Dirichlet series $D_{j}(s, \alpha)$. From (2.2), we have

$$
\begin{aligned}
D_{1}(s, \alpha)= & D_{1}(s)-\sum_{k=1}^{N} \frac{(-1)^{k-1} \alpha^{k+1}}{k(k+1)} \zeta(s+k)+\alpha \zeta^{\prime}(s)-2 \alpha \gamma \zeta(s) \\
& +O\left(\sum_{n=1}^{\infty} \frac{1}{n^{\Re s+N+1}}\right)
\end{aligned}
$$

for any $N$. The last sum converges for $\Re s>-N$. Since $N$ can be taken arbitrarily large, $D_{1}(s, \alpha)$ can be continued to the whole complex plane C. In particular, we have

$$
D_{1}(s, \alpha)=D_{1}(s)+\alpha \zeta^{\prime}(s)-2 \alpha \gamma \zeta(s)-\frac{\alpha^{2}}{2} \zeta(s+1)+O(1)
$$

for $\Re s>-1$, whose Laurent expansion of $D_{1}(s, \alpha)$ at $s=1$ is given by

$$
D_{1}(s, \alpha)=\frac{1 / 2-\alpha}{(s-1)^{2}}+\frac{\gamma(1-2 \alpha)+1 / 4}{s-1}+O(1) \text {. }
$$

Comparing (6.6) with (6.4), we have

$$
D_{1}(s, \alpha)-I_{1}(s)=-\frac{B_{1}(\alpha)}{(s-1)^{2}}-\frac{2 \gamma B_{1}(\alpha)}{s-1}+O(1),
$$

at $s=1$; in particular, we can see that $D_{1}(s, 1 / 2)-L_{1}(s)$ is holomorphic at $s=1$.

Similarly we can see that $D_{2}(s, \alpha)-D_{2}(s)$ can be continued analytically to the half plane $\Re s>-2 / 3$ as a meromorphic function. More precisely, by using (3.2), we have

$$
\begin{aligned}
D_{2}(s, \alpha)= & D_{2}(s)+2 \alpha D_{1}^{\prime}(s)-4 \alpha \gamma D_{1}(s)-\alpha^{2} D_{1}(s+1) \\
& +\alpha^{2} \zeta^{\prime \prime}(s)-4 \alpha^{2} \gamma \zeta^{\prime}(s)+4 \alpha^{2} \gamma^{2} \zeta(s)-\alpha^{3} \zeta^{\prime}(s+1) \\
& +2 \alpha^{3} \gamma \zeta(s+1)+K(s),
\end{aligned}
$$

where $K(s)$ is holomorphic in $\Re s>-2 / 3$, from which we have

$$
\begin{aligned}
D_{2}(s, \alpha)= & D_{2}(s)+\frac{2 \alpha^{2}-2 \alpha}{(s-1)^{3}}+\frac{\left(4 \alpha^{2}-4 \alpha\right) \gamma-\alpha / 2}{(s-1)^{2}} \\
& +\frac{\left(4 \alpha^{2}-4 \alpha\right) \gamma^{2}-\alpha \gamma}{s-1}+O(1)
\end{aligned}
$$


at $s=1$. Combining (6.5) and (6.9), we find that

$$
\begin{aligned}
D_{2}(s, \alpha)= & I_{2}(s)+\frac{2 B_{2}(\alpha)}{(s-1)^{3}}+\frac{4 B_{2}(\alpha) \gamma-1 / 2 B_{1}(\alpha)}{(s-1)^{2}} \\
& +\frac{4 B_{2}(\alpha) \gamma^{2}-B_{1}(\alpha) \gamma}{s-1}+O(1)
\end{aligned}
$$

Let $\beta$ and $\beta^{\prime}$ be zeros of $B_{2}(x)$. Then, from the above formula, we can see that $\left[D_{2}(s, \beta)+D_{2}\left(s, \beta^{\prime}\right)\right] / 2-I_{2}(s)$ is holomorphic at $s=1$. It is also clear from (6.8) that this difference is holomorphic at $s=3 / 2$.

Acknowledgments. The authors are grateful to the referee for a careful reading and useful comments.

\section{REFERENCES}

1. X. Cao, J. Furuya, Y. Tanigawa and W. Zhai, On the differences between two kinds of mean value formulas of number-theoretic error terms, Int. J. Num. Th. 10 (2014), 1143-1170.

2. X. Cao and W. Zhai, On the mean square of the error term for the asymmetric two-dimensional divisor problems (II), Monatsh. Math. 160 (2010), 115-142.

3. J. Furuya, On the average orders of the error term in the Dirichlet divisor problem, J. Num. Th. 115 (2005), 1-26.

4. J. Furuya, Y. Tanigawa and W. Zhai, Dirichlet series obtained from the error term in the Dirichlet divisor problem, Monatsh. Math. 160 (2010), 385-402.

5. M.N. Huxley, Exponential sums and lattice points III, Proc. Lond. Math. Soc. 87 (2003), 591-609.

6. A. Ivić, The Riemann Zeta-function, Theory and applications, Reprint of the 1985 original (John Wiley \& Sons, New York), Dover Publications, Inc., Mineola, NY, 2003.

7. , On the integral of the error term in the Dirichlet divisor problem, Bull. Sci. Math. Nat. Sci. Math. 25 (2000), 29-45.

8. A. Ivić and P. Sargos, On the higher moments of the error term in the divisor problem, Illinois J. Math. 51 (2007), 353-377.

9. Y.K. Lau and K.M. Tsang, Mean square of the remainder term in the Dirichlet divisor problem, J. Th. Nombres Bord. 7 (1995), 75-92.

10. On the mean square formula of the error term in the Dirichlet divisor problem, Math. Proc. Camb. Phil. Soc. 146 (2009), 277-287.

11. E. Preissmann, Sur la moyenne quadratique du terme de reste du problème du cercle, C.R. Acad. Sci. Paris 306 (1988), 151-154.

12. R. Sitaramachandrarao, An integral involving the remainder term in the Piltz divisor problem, Acta Arith. 48 (1987), 89-92. 
13. E.C. Titchmarsh, The theory of the Riemann Zeta-function (revised by D.R. Heath-Brown), Clarendon Press, Oxford 1986.

14. K.-C. Tong, On divisor problems III, Acta Math. Sinica 6 (1956), 515-541 (in Chinese).

15. K.M. Tsang, Higher-power moments of $\Delta(x), E(t)$ and $P(x)$, Proc. Lond. Math. Soc. 65 (1992), 65-84.

16. G.F. Voronoï, Sur un problème du calcul des fonctions asymptotiques, J. reine angew. Math. 126 (1903), 241-282.

17. , Sur une fonction transcendante et ses applications à la sommation de quelques séries, Ann. École Norm. 21 (1904), 207-267, 459-533.

18. W. Zhai, On higher-power moments of $\Delta(x)$ II, Acta Arith. 114 (2004), $35-54$.

19. $263-281$. , On higher-power moments of $\Delta(x)$ III, Acta Arith. 118 (2005),

Department of Mathematics and Physics, Beijing Institute of PetroChemical Technology, Beijing 102617, P.R. China

Email address: caoxiaodong@bipt.edu.cn

Department of Integrated Human Sciences (Mathematics), Hamamatsu University School of Medicine, Handayama 1-20-1, Higashi-ku, Hamamatsu city, SHIZUOKA, 431-3192, JAPAN

Email address: jfuruya@hama-med.ac.jp

Graduate School of Mathematics, Nagoya University, Chikusaku, Nagoya 464-8602, JAPAN

Email address: tanigawa@math.nagoya-u.ac.jp

Department of Mathematics, China University of Mining and Technology, BeiJing 100083, P.R. China

Email address: zhaiwg@hotmail.com 